\title{
Evaluación en farmacias comunitarias del estado nutricional de mayores de 65 años
}

\author{
Inés Mera-Gallego ${ }^{1,6}$, José Antonio Fornos-Pérez ${ }^{2,6,7}$, José Andrés Ferrer-Úbedaa ${ }^{3,7}$, Antonio Ruiz-Lara ${ }^{4,7}$, \\ Rocío Mera-Gallego ${ }^{5,6,7}$, Patricia García-Rodríguez ${ }^{2,6}$, Miriam Barreiro-Juncal ${ }^{5,6}$, N. Floro Andrés-Rodríguez ${ }^{5,6,7}$ \\ 1. FC en Maella (Zaragoza). 2. FC en Cangas do Morrazo (Pontevedra). 3. FC en Valencia. 4. FC en Cuenca. 5. FC en Vigo. 6. Grupo Berbés de \\ Investigación y Docencia. 7. Grupo de Diabetes de SEFAC.
}

\section{PALABRAS CLAVE}

Mayores, malnutrición, riesgo, diabetes, farmacia comunitaria

\section{ABREVIATURAS}

CP: circunferencia de la pantorrilla DE: desviación estándar FEDE: Federación Española de Diabetes

IC: indice de confianza IMC: índice de masa corporal m: media

MNA: Mini Nutritional Assessment

PNT: procedimiento normalizado de trabajo SEFAC: Sociedad Española de Farmacia Familiar y Comunitaria SENPE: Sociedad Europea de Nutrición Parenteral y Enteral

SEGG: Sociedad Española de Geriatría y Gerontología

\section{KEYWORDS}

Aged, nutritional deficiency, risk, diabetes, community pharmacy

\section{RESUMEN}

Objetivos: Evaluar el estado nutricional de mayores de 65 años con diabetes, que acuden a la farmacia comunitaria y compararlo con el de personas no diabéticas.

Método: Estudio observacional-transversal en farmacias españolas por farmacéuticos socios de SEFAC, de 9 de noviembre a 9 de diciembre de 2015. Criterios de Inclusión: $\geq 65$ años, en dos grupos: diabéticos y no diabéticos. Variables: características antropométricas, altura suelo-rodilla $(\mathrm{cm})$, circunferencia braquial $(\mathrm{cm})$, circunferencia de la pantorrilla $(\mathrm{cm})$, diabetes $(\mathrm{SÍ} / \mathrm{NO})$ y estado nutricional (cuestionario Mini Nutritional Assessment).

Resultados: 1.078 mayores, 652 (60,5\%) mujeres de 75,5 $\pm 7,4$ años, 461 (42,8\%) diabéticos. 73,4\% con sobrepeso/obesidad. IMC $=28,6 \pm 4,8$ sin diferencias significativas entre sexos, mayor en diabéticos $(29,0 \pm 4,8$ vs $28,3 \pm 4,8 \mathrm{p}=0,0173) .7,5 \%$ de diabéticos malnutridos frente al $6,5 \%$ de no diabéticos. $39,2 \%$ de diabéticos en riesgo de malnutrición frente al 29,8\% de no diabéticos. El porcentaje de mujeres en riesgo de malnutrición y/o malnutrición es superior al de hombres $(46,3 \%$ vs 32,4\% p<0,0001). Mayor en mujeres diabéticas frente no diabéticas $(52,4 \%$ vs 39,3\% $\mathrm{p}=0,0075) .73,1 \%$ de diabéticos consideran que tienen problemas de nutrición frente al 80,7\% de los no diabéticos $\mathrm{p}=0,0132$. El 30,0\% de los diabéticos consideran mejor su estado de salud frente al 45,9\% de los no diabéticos.

Conclusiones: El 82\% de mayores de 65 años presenta malnutrición y/o riesgo de malnutrición, mayor en diabéticos que en no diabéticos y en mujeres que en hombres. 3 de cada 4 mayores de 65 años tienen sobrepeso u obesidad, mayor en hombres que en mujeres, y entre diabéticos que en no diabéticos.

\section{Assessment of the nutritional condition of over 65s in community pharmacies}

\section{ABSTRACT}

Objectives: To assess the nutritional condition of over 65 s with diabetes that go community pharmacies, and compare it to individuals without diabetes.

Method: Observational-transversal study in Spanish pharmacies by pharmacists that are SEFAC partners, from November 9 to December 9 2015. Inclusion criteria: $\geq 65$ years old, in two groups: diabetic and non-diabetic. Variables: anthropometric characteristics, floor-knee distance $(\mathrm{cm})$, brachial circumference $(\mathrm{cm})$, calf circumference $(\mathrm{cm})$, diabetes (YES/NO) and nutritional condition (Mini Nutritional Assessment).

Results: 1078 elderly; 652 (60.5\%) women, 75.5 \pm 7.4 years old; 461 (42.8\%) diabetic. 73.4\% overweight/obese. $\mathrm{BMI}=28.6 \pm 4.8$ with no significant differences between genders; higher in diabetics ( $29.0 \pm 4.8$ vs. $28.3 \pm 4.8 p=0.0173)$. $7.5 \%$ of diabetics presented symptoms of malnutrition vs. $6.5 \%$ of non-diabetics. $39.2 \%$ of diabetics at risk of malnutrition vs. $29.8 \%$ of non-diabetics. There were more women at risk of malnutrition and/or presenting symptoms of malnutrition than men (46.3\% vs. 32.4\% $\mathrm{p}>0.0001)$. Higher in diabetic women than in non-diabetic women (52.4\% vs. 39.3\% $\mathrm{p}=0.0075) .73 .1 \%$ of diabetics believe they suffer from nutritional issues vs. $80.7 \%$ of non-diabetics $p=0.0132 .30 .0 \%$ of diabetics believe their health is better vs. $45.9 \%$ of non-diabetics.

Conclusions: Eighty-two percent of over 65s present symptoms of malnutrition and/or are at risk of malnutrition; the rate is higher among diabetics than non-diabetics and among women than men. Three in every four over 65s are overweight or obese; the rate is higher in men than women and in diabetics than non-diabetics.

Contribución a la autoría: IM-G y JAF-P contribuyeron al diseño del estudio, desarrollo de la investigación, análisis de los resultados, tratamiento estadistico, redacción del manuscrito y revisión del contenido final. JAF-U, AR-L, PG-R y MB-J al desarrollo de la investigación y la revisión del contenido final. RM-G contribuyó al diseño del estudio, desarrollo de la investigación, análisis de los resultados, redacción del manuscrito y revisión del contenido final. NFA-R contribuyó al diseño del estudio, análisis de los resultados, tratamiento estadistico, redacción del manuscrito y revisión del contenido final.

Cite este artículo como: Mera-Gallego I, Fornos-Pérez JA, Ferrer-Úbeda JA, Ruiz-Lara A, Mera-Gallego R, Garcia-Rodriguez P, Barreiro-Juncal M, Andrés-Rodriguez NF. Evaluación en farmacias comunitarias del estado nutricional de mayores de 65 años. Farmacéuticos Comunitarios. 2017 Jun 30; 9(2):5-23. doi:10.5672/FC.2173-9218.(2017Nol9).002.02

Correspondencia: Inés Mera-Gallego (inesmg@grupoberbes.es)

ISSN 1885-8619 @SEFAC (Sociedad Española de Farmacia Familiar y Comunitaria). Todos los derechos reservados. 


\section{Introducción}

El envejecimiento es un proceso natural del ser humano que cada persona experimenta con diferente intensidad. En la actualidad es difícil establecer el comienzo de esta etapa de vida en función exclusiva de la edad. No obstante, se considera que una persona es adulta mayor a partir de los 60-65 años [1]. España, junto a Italia y Japón, encabeza este proceso de envejecimiento a nivel mundial estimándose que en el año 2050 cerca del 35\% de nuestra población superará los 65 años [2].

Durante esta etapa se producen modificaciones importantes en la composición corporal (disminución de la masa magra, aumento de la masa grasa y disminución de la masa ósea), en el funcionamiento del aparato digestivo (reducción de la función motora, cambios en la secreción, alteración en mecanismos reguladores, etc.), en la actividad metabólica (disminución del metabolismo basal, alteración del metabolismo de hidratos de carbono, disminución del de lípidos y proteínas) y en otros aspectos fisiológicos (pérdidas dentarias, disminución de las papilas gustativas, disminución de la percepción del olfato, pérdida de agudeza visual, etc.) [3,4].

Todos estos cambios generan una ingesta inadecuada de alimentos, un deterioro o alteración en la capacidad para digerir, absorber y metabolizar nutrientes o una alteración de la utilización de ellos, provocando así que un gran porcentaje de adultos mayores estén en riesgo de malnutrición o malnutridos [5].

La malnutrición se considera un síndrome geriátrico que podría definirse como una alteración en las etapas de la nutrición, tanto por déficit que conlleva a la desnutrición; como por exceso que trae consigo la obesidad, es decir, es el resultado de un desequilibrio entre las necesidades corporales y la ingesta de nutrientes $[6,7]$. Ésta produce alteraciones del estado inmunitario, complicaciones de patologías existentes, etc. Es decir, un aumento de morbi-mortalidad. Su prevalencia oscila entre un 3-5\% mayoritariamente, aunque llega hasta un $44,7 \%$ en personas no institucionalizadas [8], un 40\% en hospitales $[9,10]$ y más de un $50 \%$ en personas ingresadas en instituciones o residencias [11].
Sin embargo, la mayoría de los problemas nutricionales encontrados en este colectivo se podrían solucionar si se llevasen a cabo planes estructurados de detección de malnutrición y evaluaciones nutricionales tempranas con la intención de intervenir en mejorar dicho estado nutricional, mejorando así su calidad de vida $[12,13]$.

En los mayores diabéticos, con el envejecimiento se produce de manera fisiológica una pérdida de sensibilidad a la insulina. El sobrepeso y la obesidad son frecuentes en la población mayor con diabetes; además, se produce una redistribución de la grasa corporal, con un incremento de la adiposidad visceral. Otros factores que contribuyen a la resistencia insulínica son las modificaciones en el estilo de vida que se dan en individuos de edad avanzada (reducción de la actividad física, cambios en la dieta con una mayor ingesta de alimentos ricos en grasas saturadas, ricas en azúcares simples y pobres en carbohidratos complejos), y un mayor consumo de fármacos que interfieren el metabolismo hidrocarbonado. De esta manera, los mayores con diabetes son pacientes con alto riesgo de desnutrición, llegando a padecerla, según algunos estudios, un 50\% de los mismos [14].

La evaluación del estado nutricional no solo permite identificar o diagnosticar a los grupos vulnerables con riesgo de malnutrición sino también sirve para controlar la eficacia de tratamientos nutricionales y/o farmacológicos, establecer valores de referencias y desarrollar programas de prevención y/o tratamientos en nutrición y salud.

La valoración nutricional geriátrica siempre está precedida por un cribado nutricional, cuyo objetivo es detectar sujetos con riesgo de malnutrición o malnutridos que ha de ser sometidos a una valoración completa para establecer el diagnóstico nutricional e implementar un plan de actuación personalizado. El cribado nutricional periódico es, por tanto, fundamental para poder intervenir y mejorar el estado de salud y la calidad de vida de los usuarios y la farmacia comunitaria es el centro sanitario que la población tiene más cercano y donde puede dirigirse sin cita previa, de modo que la accesibilidad y la cercanía influye en la alta frecuencia de visitas y en muchas consultas de salud, Además, la formación sanitaria del farmacéutico y su formación complementaria en nutrición, hacen de él el profesional más adecuado para llevarlo a cabo.

La mayoría de los estudios nutricionales en ancianos se han llevado a cabo en instituciones cerradas (residencias $u$ hospitales) [9-11] y muy pocos en ancianos no institucionalizados, los cuales también tienen un riesgo elevado de malnutrición [8]. En farmacia comunitaria existen algunas experiencias como el Plenufar 3 dirigido a personas mayores de 65 años, que se llevó a cabo en 2005 en farmacias, centros de personas mayores y otros lugares como asociaciones de amas de casa, centros cívicos, etc. Entre sus objetivos se incluian el asesoramiento a las personas mayores en hábitos dietéticos saludables y valoración del estado nutricional de personas mayores de 65 años [15]. Se encuentran también comunicaciones a congresos sobre valoración nutricional de mayores en farmacias comunitarias [16-19], pero muy pocos artículos publicados y no de actualidad [20,21].

Con el presente trabajo, el Grupo de Diabetes de SEFAC en colaboración con la Federación Española de Diabetes (FEDE) pretende documentar la idoneidad del farmacéutico desde la farmacia comunitaria para realizar cribados nutricionales en las farmacias comunitarias españolas.

\section{Objetivos}

Objetivo principal

Evaluar el estado nutricional de las personas mayores de 65 años con diabetes, que acuden a la farmacia comunitaria comparando personas con diabetes y sin diabetes.

\section{Objetivos secundarios}

- Determinar las características antropométricas de los mayores de 65 años, en función del sexo y de ser o no diabéticos.

- Analizar las respuestas al cuestionario Mini Nutritional Assessment (MNA) y su puntuación global, según sexo y padecer o no diabetes.

- Analizar las diferencias en riesgo de malnutrición en función del sexo y de ser o no diabético. 


\section{Métodos}

\section{Diseño y ámbito}

Estudio observacional transversal realizado en farmacias comunitarias españolas, desde el 9 de noviembre hasta el 9 de diciembre de 2015.

\section{Sujetos}

Criterios de inclusión: personas de 65 años o más que acuden a la farmacia, con capacidad suficiente para contestar y que consientan hacerlo.

Criterios de exclusión: menores de 65 años, no capacitadas para cumplimentar el cuestionario o que se nieguen a participar.

\section{Tamaño muestral}

El universo muestral son los usuarios de las farmacias comunitarias españolas cuyos farmacéuticos son socios de SEFAC. Para el cálculo del tamaño muestral se tuvo en cuenta que la población española es de 46.449.565 habitantes y que los individuos de 65 años y más son 5.388.895 [22]. Para conseguir una precisión del 3,0\% en la estimación de una proporción mediante un intervalo de confianza asintótico normal con corrección para poblaciones finitas al 95\% bilateral, asumiendo que la proporción esperada es del 50\% se estimó necesario incluir 1.060 unidades experimentales totales en el estudio. El porcentaje de diabéticos diagnosticados es del 7\%, por lo que la población de diabéticos españoles es de 3.080.000 [23]. Por lo tanto, para conseguir una precisión del 1,0\% en la estimación de una proporción mediante un intervalo de confianza asintótico normal con corrección para poblaciones finitas al 95\% bilateral, asumiendo una proporción esperada del 50\%, y considerando unas pérdidas del 20\%, era necesario incluir 462 diabéticos en el estudio.

\section{Variables}

Edad (años), sexo (H/M), peso $(\mathrm{kg})$, talla $(\mathrm{m})$, índice de masa corporal (IMC) $\left(\mathrm{kg} / \mathrm{m}^{2}\right)$, circunferencia de la pantorrilla (CP) $(\mathrm{cm})$, diabetes (Sí/No), y las preguntas del cuestionario Mini Nutritional Assessment (MNA) [24]: $<17$ puntos, desnutrición; 17-23,5 puntos, riesgo de desnutrición; 24-30 puntos, estado nutricional normal.

El MNA es el cuestionario con mayor aceptación, validez y utiliza- ción a nivel mundial para detectar situaciones de riesgo de malnutrición o de malnutrición sin necesidad de recurrir a técnicas complejas [25]. Aunque puede ser autoadministrado, en este estudio fue administrado por los farmacéuticos comunitarios. Se utilizó la forma completa, recomendada por la Sociedad Española de Nutrición Parenteral y Enteral (SENPE) para ser usada en población mayor de 65 años [25]. Consta de dos partes: la primera contiene 6 ítems a partir de los cuales se obtiene una puntuación $(0,0,5,1,2$ o 3) que permite clasificar a los ancianos como bien nutridos, en riesgo de malnutrición o malnutridos. La segunda parte está formada por 12 items referentes a parámetros antropométricos, cuestiones referidas a la ingesta de alimentos, a la valoración general de la salud del paciente y a la percepción que tiene el propio paciente sobre su estado nutricional y de salud. Una vez completada se obtiene una puntuación total que permite conocer si el sujeto se encuentra bien nutrido, en riesgo de malnutrición o malnutrido.

\section{Procedimiento}

Se informó del proyecto a los socios de SEFAC y se permitió la inscripción de 100 socios de toda España, a los cuales se envió el material impreso: cuestionario MNA, cartel de captación, protocolo de trabajo, procedimientos normalizados de trabajo (PNT) para las determinaciones antropométricas, consejos nutricionales y decálogo de consejos higiénico-dietéticos para entregar a los mayores participantes (anexos 1-7).

1. Ofrecimiento a los usuarios de 65 años o más, que acuden a la farmacia entre 9 de noviembre y 9 de diciembre de 2015.

2. Realizar las determinaciones antropométricas.

3. Cumplimentación de la encuesta.

4. Intervención del farmacéutico: información al paciente de su estado nutricional, su significado y recordatorio, entregándole información escrita, de la importancia de los hábitos higiénico-dietéticos saludables. En caso de desnutrición, derivación al médico con copia del resultado del MNA.

5. Los resultados se registraron de manera anónima en un formulario desarrollado ad hoc disponible en la página web de SEFAC.
Análisis estadístico

Se utilizó el programa G-Stat ${ }^{\circledR}$ 2.0 (Glaxo Smithkline). Los datos cualitativos se expresan como porcentajes y los cuantitativos como media \pm desviación estándar. Los límites de confianza se calculan al 95\% (IC). Se utilizó el test de chi-cuadrado para comparación de proporciones o la prueba de Fisher en el caso de muestras pequeñas. Para comparar medias se empleó la T-Student para variables que siguen una distribución normal (Test de Kolmogorov con las correcciones de Lilliefors) y los test no paramétricos U-Mann-Withney o Wilcoxon, para variables sin distribución normal. Las correlaciones se determinaron mediante la $r$ de Pearson o Rho de Spearman según fueran o no variables paramétricas. La significación estadística se fijó en $\mathrm{p}<0,05$.

\section{Consideraciones éticas}

El estudio cumple los criterios exigidos por el Comité de Etica de Investigación de la Universidad CEU San Pablo de Madrid. Los participantes firmaron un documento de consentimiento informado sobre el estudio a realizar. Aunque en la hoja de registro se recogían los datos personales del mayor participante, el volcado en la web de SEFAC se realizaba de forma anónima.

\section{Resultados}

Se inscribieron 139 socios, de los que se admitieron los 100 primeros. Completaron envíos de manera correcta, dentro del plazo establecido, 72 farmacéuticos pertenecientes a todas las comunidades autónomas excepto Ceuta y Melilla (tabla 1). Se recibieron 1.095 formularios enviados en plazo, de los que 1.078 estaban correctamente cumplimentados.

De los 1.078 usuarios entrevistados, 652 (60,5\%) eran mujeres. 461 (42,8\%) eran diabéticos. En la tabla 2 se muestran los datos antropométricos de la muestra. El 77,4\% de los entrevistados tenía sobrepeso u obesidad, siendo mayor entre los hombres $(80,8 \%)$ que entre las mujeres $(74,6 \%)$ (figura 1$)$.

Los resultados del cuestionario MNA, con el número y porcentaje de entrevistados para cada posible respuesta a los ítems se presentan en la tabla 3. En la tabla 4 su distribución y la del IMC según la estratificación etaria. 


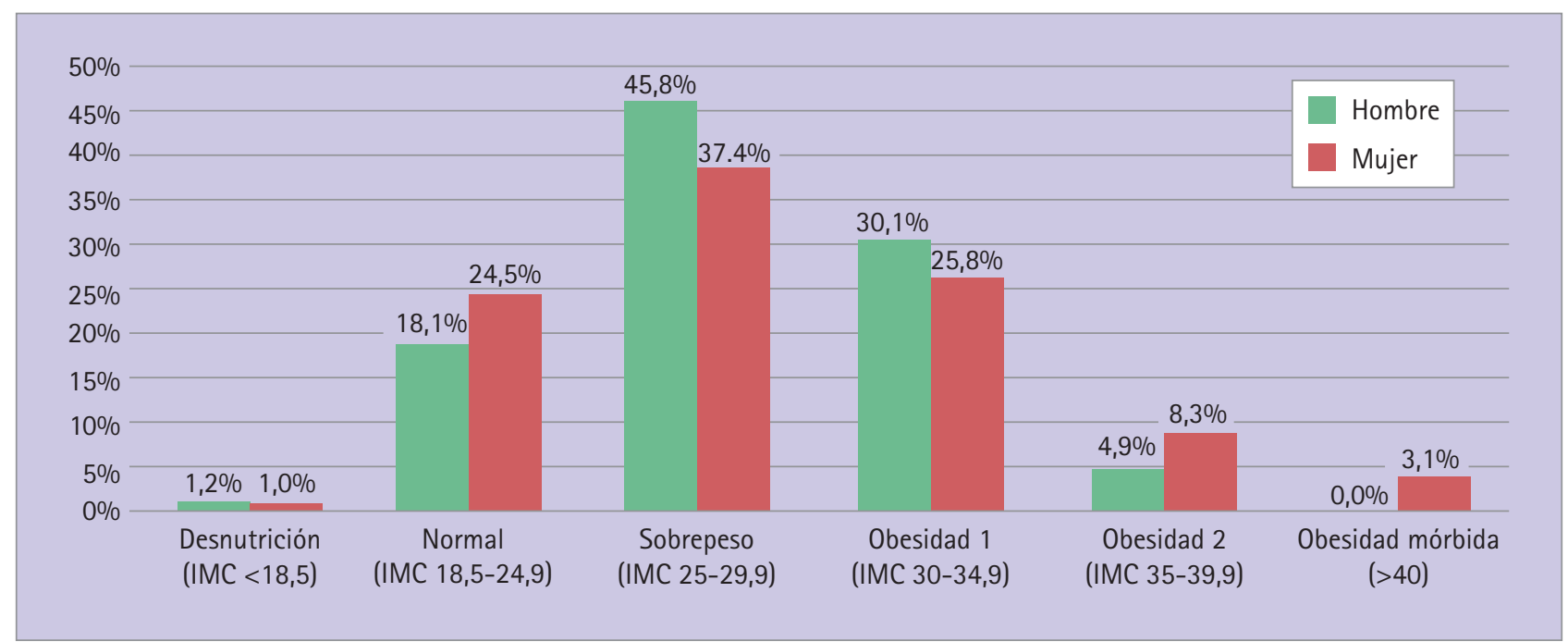

Figura 1 Distribución de la muestra en función del IMC y sexo

Tabla 1 Número de farmacias participantes distribuidas por comunidad autónoma

\begin{tabular}{|l|c|c|}
\hline Comunidad autónona & No de farmacia inscritas & No de farmacias participantes \\
\hline Andalucia & 15 & 9 \\
\hline Aragón & 3 & 3 \\
\hline Asturias & 2 & 2 \\
\hline Islas Baleares & 2 & 2 \\
\hline Canarias & 5 & 3 \\
\hline Cantabria & 2 & 2 \\
\hline Castilla-La Mancha & 5 & 4 \\
\hline Castilla y León & 5 & 4 \\
\hline Cataluña & 16 & 9 \\
\hline Extremadura & 2 & 2 \\
\hline Galicia & 9 & 5 \\
\hline La Rioja & 1 & 1 \\
\hline Comunidad de Madrid & 13 & 9 \\
\hline Navarra & 2 & 2 \\
\hline Pais Vasco & 4 & 4 \\
\hline Región de Murcia & 3 & 3 \\
\hline Comunidad Valenciana & 11 & 8 \\
\hline Total & 100 & 72 \\
\hline
\end{tabular}

La puntuación total del MNA fue de 23,6 $\pm 4,3$ significativamente mayor en hombres que en mujeres $(24,3 \pm 3,7$ vs $23,3 \pm 4,5 \mathrm{p}<0,0001)$, tanto en diabéticos $(23,9 \pm 3,6$ vs $22,8 \pm 4,3 \mathrm{p}<0,01)$ como en no diabéticos $(24,5 \pm 4,3$ vs $23,8 \pm 4,2 \mathrm{p}<0,01)$.

La puntuación de los entrevistados con $\mathrm{CP}<31(19,6 \pm 5,6)$ es significativamente inferior a la puntua- ción de los entrevistados con $\mathrm{CP}>31$ $(24,6 \pm 3,3)(p<0,001)$. En la tabla 5 se muestran los resultados en función del estado nutricional y el CP.

En la tabla 6 se presenta la valoración nutricional en función del MNA y sexo. El porcentaje de mujeres en situación de riesgo de malnutrición y malnutrición fue superior al de los hombres: $46,3 \%$ vs 32,4\% $\mathrm{p}<0,0001$.

En la comparación entre los encuestados con y sin diabetes, encontramos que las únicas diferencias estadísticamente significativas, desde el punto de vista antropométrico, entre ambos grupos, fueron el IMC, mayor en diabéticos $(29,0 \pm 4,8)$ que en no diabéticos $(28,3 \pm 4,8) \mathrm{p}=0,0173$, y el porcentaje de individuos con $\mathrm{CP}<31$ : diabéticos 108 (23,7\%) vs no diabéticos $88(14,4 \%) \mathrm{p}<0,001$ (tabla 7). El porcentaje de individuos con sobrepeso u obesidad fue también mayor en diabéticos $369(80,1 \%)$ que en no diabéticos 689 (74,7\%).

El porcentaje de diabéticos en situación de riesgo de malnutrición y malnutrición es superior al de los no diabéticos 216 (46,7\%) vs 224 (36,3\%) $\mathrm{p}=0,0022$ (tabla 6). Mayor entre las mujeres que entre los hombres 139 $(52,4 \%)$ vs $76(39,3 \%) \mathrm{p}=0,0075$ (tabla 6).

Tabla 2 Datos antropométricos de la muestra

\begin{tabular}{|l|c|c|c|c|c|c|}
\multicolumn{1}{|c|}{ Sexo } & $n(\%)$ & $\begin{array}{c}\text { Edad } \\
m \pm D E(m i n-m a ́ x)\end{array}$ & $\begin{array}{c}\text { Peso } \\
m \pm D E\end{array}$ & $\begin{array}{c}\text { Talla } \\
m \pm D E\end{array}$ & $\begin{array}{c}\text { IMC } \\
m \pm D E\end{array}$ & $\begin{array}{c}\text { Con diabetes } \\
n(\%)\end{array}(\%$ por sexo) \\
\hline Hombre & $426(39,5)$ & $75,6 \pm 7,3(65-95)$ & $79,1 \pm 13,1$ & $166,9 \pm 11,5$ & $28,3 \pm 3,9$ & $196(18,2)(46,0)$ \\
\hline Mujer & $652(60,5)$ & $75,4 \pm 7,5(65-99)$ & $69,7 \pm 13,6$ & $155,6 \pm 9,3$ & $28,7 \pm 5,3$ & $265(24,6)(40,6)$ \\
\hline Total & $1.078(100,0)$ & $75,5 \pm 7,4$ & $73,5 \pm 13,2$ & $159,5 \pm 10,2$ & $\mathbf{2 8 , 6 \pm 4 , 8}$ & $\mathbf{4 6 1}(\mathbf{4 2 , 8 )}$ \\
\hline
\end{tabular}

IMC: indice de masa corporal. 


\begin{tabular}{|c|c|c|c|c|c|}
\hline \multicolumn{2}{|c|}{ Preguntas del MNA } & n (\%) & n (\%) & n (\%) & n (\%) \\
\hline \multirow{2}{*}{ A } & \multirow{2}{*}{$\begin{array}{l}\text { ¿Ha perdido el apetito? ¿Ha comido menos } \\
\text { por falta de apetito, problemas digestivos, } \\
\text { dificultades de masticación o deglución en } \\
\text { los últimos } 3 \text { meses? }\end{array}$} & Anorexia grave & Anorexia moderada & Sin anorexia & \\
\hline & & $31(2,9)$ & $212(19,7)$ & $835(77,5)$ & \\
\hline \multirow{2}{*}{ B } & \multirow{2}{*}{ Pérdida reciente de peso (<3 meses) } & $>3 \mathrm{Kg}$ & No lo sabe & $1-3 \mathrm{Kg}$ & No pérdida \\
\hline & & $87(8,1)$ & $118(11,0)$ & $160(14,8)$ & $713(66,1)$ \\
\hline \multirow{2}{*}{ C } & \multirow{2}{*}{ Movilidad } & De cama al sillón & Autonomía en el interior & Sale del domicilio & \\
\hline & & $44(4,1)$ & $63(5,8)$ & $971(90,1)$ & \\
\hline \multirow{2}{*}{ D } & \multirow{2}{*}{$\begin{array}{l}\text { ¿Ha tenido una enfermedad aguda o } \\
\text { situación de estrés psicológico en los } \\
\text { últimos } 3 \text { meses? }\end{array}$} & Si & No & & \\
\hline & & $278(25,8)$ & $800(74,2)$ & & \\
\hline \multirow[t]{2}{*}{$\mathrm{E}$} & \multirow[t]{2}{*}{ Problemas neuropsicológicos } & $\begin{array}{l}\text { Demencia o } \\
\text { depresión grave }\end{array}$ & Moderada & $\begin{array}{l}\text { Sin problemas } \\
\text { psicológicos }\end{array}$ & \\
\hline & & $38(3,5)$ & $256(23,8)$ & $784(72,7)$ & \\
\hline \multirow{2}{*}{$\mathrm{F}$} & \multirow{2}{*}{ IMC } & $<19$ & $19-21$ & $21-23$ & $>23$ \\
\hline & & $24(2,2)$ & $43(4,0)$ & $78(7,2)$ & $933(86,6)$ \\
\hline \multirow{2}{*}{ G } & \multirow{2}{*}{$\begin{array}{l}\text { ¿El paciente vive independiente en su } \\
\text { domicilio? }\end{array}$} & Si & No & & \\
\hline & & $884(82,0)$ & $194(18,0)$ & & \\
\hline \multirow{2}{*}{$\mathrm{H}$} & \multirow{2}{*}{ ¿Toma más de 3 medicamentos al día? } & Si & No & & \\
\hline & & $748(69,4)$ & $330(30,6)$ & & \\
\hline \multirow{2}{*}{1} & \multirow{2}{*}{ ¿Úlceras o lesiones cutáneas? } & Si & No & & \\
\hline & & $115(10,7)$ & $963(89,3)$ & & \\
\hline \multirow{3}{*}{ J } & \multirow{2}{*}{ ¿Cuántas comidas completas toma al dia? } & 1 comida & 2 comidas & 3 comidas & \\
\hline & & $245(22,8)$ & $420(39,0)$ & $410(38,1)$ & \\
\hline & ¿Consume el paciente: & sí & NO & & \\
\hline $\mathrm{K} 1$ & Productos lácteos al menos una vez al día? & $935(86,7)$ & $143(13,3)$ & & \\
\hline $\mathrm{K} 2$ & $\begin{array}{l}\text { Huevos o legumbres } 1 \text { o } 2 \text { veces a la } \\
\text { semana? }\end{array}$ & $949(88,1)$ & $129(11,9)$ & & \\
\hline K3 & Carne, pescado o aves, diariamente? & $807(74,9)$ & $271(25,1)$ & & \\
\hline \multirow{2}{*}{$\mathrm{L}$} & \multirow{2}{*}{$\begin{array}{l}\text { ¿Consume frutas o verduras al menos } 2 \\
\text { veces al dia? }\end{array}$} & Si & No & & \\
\hline & & $819(76,0)$ & $259(24,0)$ & & \\
\hline \multirow{2}{*}{ M } & \multirow{2}{*}{$\begin{array}{l}\text { ¿Cuántos vasos de agua u otros líquidos } \\
\text { toma al día? }\end{array}$} & $<3$ & $3-5$ & $>5$ & \\
\hline & & $168(15,6)$ & $473(43,9)$ & $437(40,5)$ & \\
\hline \multirow[t]{2}{*}{$\mathrm{N}$} & \multirow[t]{2}{*}{ ¿Forma de alimentarse? } & Necesita ayuda & Solo con dificultad & Solo sin dificultad & \\
\hline & & $30(2,8)$ & $66(6,1)$ & $982(91,1)$ & \\
\hline 0 & $\begin{array}{l}\text { ¿Se considera el paciente que está bien } \\
\text { nutridn? }\end{array}$ & Malnutrición grave & $\begin{array}{l}\text { No lo sabe o } \\
\text { malnutrición moderada }\end{array}$ & $\begin{array}{l}\text { Sin problemas de } \\
\text { nutrición }\end{array}$ & \\
\hline & & $17(1,5)$ & $227(21,1)$ & $834(77,4)$ & \\
\hline & En comparación con las personas de su & Peor & No lo sabe & Igual & Mejor \\
\hline$P$ & $\begin{array}{l}\text { estado de salud? } \\
\text { estatrater su }\end{array}$ & $157(14,6)$ & $124(11,5)$ & $376(34,9)$ & $421(39,1)$ \\
\hline 0 & Circunferencia hraquial (CR en $\mathrm{cm}$ ) & $<21$ & $21-22$ & $>22$ & \\
\hline U & Circunterencla braquial (CB en cm) & $57(5,3)$ & $120(11,1)$ & $901(83,6)$ & \\
\hline $\mathbf{P}$ & Circunferencia de la nantorrilla $(\mathrm{CP}$ en $\mathrm{cm}$ ) & $<31$ & $>31$ & & \\
\hline $\mathrm{K}$ & circunterencla de la pantorma (cP en cm & $196(18,2)$ & $882(81,8)$ & & \\
\hline
\end{tabular}


Tabla 4 Resultados IMC medio y MNA en función de rango de edad y sexo

\begin{tabular}{|c|c|c|c|c|c|c|c|}
\hline \multirow[b]{2}{*}{ Edad } & \multicolumn{3}{|c|}{$\begin{array}{c}\text { IMC } \\
n(m \pm D E)\end{array}$} & \multicolumn{4}{|c|}{$\begin{array}{c}\text { MNA } \\
n(m \pm D E)\end{array}$} \\
\hline & $\begin{array}{l}\text { Mujer } \\
n=652\end{array}$ & $\begin{array}{c}\text { Hombre } \\
n=426\end{array}$ & p-valor & $\begin{array}{c}\text { Total } \\
n=1078\end{array}$ & $\begin{array}{c}\text { Mujer } \\
n=652\end{array}$ & $\begin{array}{c}\text { Hombre } \\
n=426\end{array}$ & p-valor \\
\hline $\begin{array}{l}65 \text { a } 74 \\
n=525\end{array}$ & $\begin{array}{c}322 \\
(29,5 \pm 5,3)\end{array}$ & $\begin{array}{c}203 \\
(28,5 \pm 4,2)\end{array}$ & $p<0,05$ & $24,3 \pm 3,8$ & $\begin{array}{c}322 \\
(24,1 \pm 3,6)\end{array}$ & $\begin{array}{c}203 \\
(24,4 \pm 4,0)\end{array}$ & 0,3407 \\
\hline $\begin{array}{l}75 \text { a } 84 \\
n=416\end{array}$ & $\begin{array}{c}244 \\
(28,6 \pm 5,2)\end{array}$ & $\begin{array}{c}172 \\
(28,5 \pm 3,7)\end{array}$ & $p=0,892$ & $23,6 \pm 4,1$ & $\begin{array}{c}244 \\
(23,1 \pm 4,2)\end{array}$ & $\begin{array}{c}172 \\
(24,4 \pm 3,8)\end{array}$ & $<0,01$ \\
\hline \multirow[t]{2}{*}{$\begin{array}{l}\geq 85 \\
n=137\end{array}$} & $86(27,1 \pm 3,8)$ & $51(26,5 \pm 5,0)$ & $p=0,782$ & $21,2 \pm 6,0$ & $86(22,7 \pm 4,6)$ & $51(20,3 \pm 6,5)$ & $<0,05$ \\
\hline & 652 & 426 & & & 652 & 426 & \\
\hline
\end{tabular}

\begin{tabular}{r|l|c|c|c|c|}
$\begin{array}{r}\text { Tabla 5 } \\
\text { Valoración }\end{array}$ & Normal & Riesgo & Malnutrición & Total fila \\
$\begin{array}{r}\text { nutricional } \\
\text { en función de }\end{array}$ & $\mathrm{CP}>31 \mathrm{~cm}$ & $584(66,2)$ & $272(30,8)$ & $26(3,0)$ & $882(81,8)$ \\
\hline \begin{tabular}{r} 
la CP \\
\cline { 2 - 6 }
\end{tabular} & $\mathrm{CP}<31 \mathrm{~cm}$ & $54(27,6)$ & $92(46,9)$ & $50(25,5)$ & $196(18,2)$ \\
\cline { 2 - 6 } & Total columna & $638(59,2)$ & $364(33,8)$ & $76(7,1)$ & $1.078(100,0)$ \\
\hline
\end{tabular}

$\mathrm{CP}$ : circunferencia de pantorrilla.

Tabla 6 Valoración nutricional en función del MNA y sexo y MNA, sexo y la condición diabetes

\begin{tabular}{|c|c|c|c|c|c|c|c|}
\hline Sexo & & $\begin{array}{c}\text { Cribado } \\
m \pm D E\end{array}$ & $\begin{array}{c}2^{\mathrm{a}} \text { parte } \\
\mathrm{m} \pm \mathrm{DE}\end{array}$ & $\begin{array}{l}\text { MNA } \\
m \pm D E\end{array}$ & $\begin{array}{l}\text { Normal } \\
\mathrm{n}(\%)\end{array}$ & $\begin{array}{c}\text { Riesgo } \\
n(\%)\end{array}$ & $\begin{array}{c}\text { Malnutrición } \\
\mathrm{n}(\%)\end{array}$ \\
\hline Hombre & 426 & $12,4 \pm 2,1$ & $12,3 \pm 2,0$ & $24,3 \pm 3,7$ & $288(67,6)$ & $120(28,2)$ & $18(4,2)$ \\
\hline Mujer & 652 & $11,7 \pm 2,4$ & $12,0 \pm 2,5$ & $23,3 \pm 4,5$ & $350(53,7)$ & $244(37,4)$ & $58(8,9)$ \\
\hline \multirow[t]{2}{*}{ Total } & 1.078 & & & & $638(59,2)$ & $364(33,8)$ & $76(7,1)$ \\
\hline & & $p<0,0001$ & $p<0,0001$ & $p<0,0001$ & \multicolumn{3}{|c|}{$p<0,0001$} \\
\hline Diabético & 461 & $11,4 \pm 2,4$ & $11,8 \pm 2,3$ & $23,2 \pm 4,1$ & $245(53,3)$ & $181(39,2)$ & $35(7,5)$ \\
\hline No Diabético & 617 & $12,1 \pm 2,5$ & $12,0 \pm 2,4$ & $24,1 \pm 4,3$ & $393(63,7)$ & $184(29,8)$ & $40(6,5)$ \\
\hline \multirow[t]{2}{*}{ Total } & 1.078 & & & & $638(59,2)$ & $365(33,8)$ & $75(7,0)$ \\
\hline & & $p<0,001$ & $p=0,1452$ & $p<0,001$ & \multicolumn{3}{|c|}{$p=0,0022$} \\
\hline Hombre diabético & 196 & $12,2 \pm 2,0$ & $11,6 \pm 2,4$ & $23,9 \pm 3,6$ & $119(60,7)$ & $68(34,7)$ & $9(4,6)$ \\
\hline \multirow[t]{3}{*}{ Mujer diabética } & 265 & $11,6 \pm 2,5$ & $11,3 \pm 2,5$ & $22,8 \pm 4,3$ & $126(47,6)$ & $113(42,6)$ & $26(9,8)$ \\
\hline & 461 & & & & $245(53,3)$ & $181(39,2)$ & $35(7,5)$ \\
\hline & & $p<0,01$ & $P=0,1927$ & $p<0,01$ & \multicolumn{3}{|c|}{$p=0,0075$} \\
\hline
\end{tabular}

MNA: Mini Nutritional Assessment.

\begin{tabular}{|c|c|c|c|c|c|c|c|}
\hline $\begin{array}{r}\text { Tabla } 7 \\
\text { Datos }\end{array}$ & & & $\begin{array}{c}\text { Edad } \\
m \pm D E(\text { min-máx })\end{array}$ & $\begin{array}{l}\text { Peso } \\
m \pm D E\end{array}$ & $\begin{array}{c}\text { Talla } \\
m \pm D E\end{array}$ & $\begin{array}{c}\mathrm{IMC} \\
\mathrm{m} \pm \mathrm{DE}\end{array}$ & $\begin{array}{l}\mathrm{CP}<31 \\
\mathrm{n}(\%)\end{array}$ \\
\hline \multirow{3}{*}{$\begin{array}{r}\text { antropométricos } \\
\text { en función de } \\
\text { la condición } \\
\text { diabetes }\end{array}$} & Diabético & 461 & $74,8 \pm 7,1(65-94)$ & $74,3 \pm 14,4$ & $159,8 \pm 14,2$ & $29,0 \pm 4,8$ & $108(23,7)$ \\
\hline & No diabético & 617 & $76,0 \pm 7,5(65-99)$ & $72,8 \pm 14,0$ & $160,0 \pm 9,3$ & $28,3 \pm 4,8$ & $88(14,4)$ \\
\hline & & 1.078 & & & & $p=0,0173$ & $p<0,0001$ \\
\hline
\end{tabular}

CP: circunferencia de pantorrilla. 
Se encontraron diferencias significativas entre diabéticos y no diabéticos en las respuestas a las siguientes preguntas:

H. ¿Toma más de 3 medicamentos al día? La contestación SÍ es más frecuente en los diabéticos $(81,9 \%)$ que entre los no diabéticos (60,1\%), $\mathrm{p}<0,0001$.

I. ¿Úlceras o lesiones cutáneas? La contestación SÍ es más frecuente en los diabéticos $(13,4 \%)$ que entre los no diabéticos (8,7\%), $\mathrm{p}=0,0144$.

0 . ¿Se considera el paciente que está bien nutrido? (problemas nutricionales). El 73,1\% de los diabéticos consideran que no tienen problemas de nutrición frente al 80,7\% de los no diabéticos $\mathrm{p}=0,0132$.

P. En comparación con las personas de su edad, ¿cómo encuentra el paciente su estado de salud? El 16,9\% de los diabéticos consideran peor su estado de salud frente al 12,8\% de los no diabéticos. Y el 30,1\% de los diabéticos consideran mejor su estado de salud frente al 45,9\% de los no diabéticos,

\section{Discusión}

Al pretender captar una muestra representativa en España no se pudo realizar una formación presencial con todos los farmacéuticos participantes, salvo por información escrita, pudiendo existir así diferencias en la interpretación de las respuestas al cuestionario MNA, en la realización de las medidas antropométricas y en el calibrado de los distintos aparatos utilizados para la medida del peso, la talla y la distancia de talón-rodilla.

En relación a la selección de las farmacias, se intentó seguir el criterio de proporcionalidad en el territorio español para conseguir así una muestra representativa de personas mayores de 65 años que acuden a las farmacias comunitarias, pero la limitación de financiación de SEFAC limitó el reclutamiento a 100 farmacias y por tanto acotó el número de mayores participantes. A pesar de ello, consideramos que la muestra final de 1.078 personas es una muestra amplia y permite obtener una estimación fiable de la malnutrición y del riesgo de malnutrición de la población mayor en España. En este sentido, aunque la captación de participantes se hizo de manera aleatorizada, y se recalcó el rigor en su aplicación durante la formación de los farmacéuticos participantes, no se descarta, como en todos los estudios de este tipo, un mínimo sesgo en la selección. La presencia del cartel anunciador también pudo estimular el interés de participantes que ya consideraban tener problemas.

Otra limitación existente es relativa al resultado de la pregunta $\mathrm{C}$ del MNA, sobre movilidad, ya que en el presente estudio tuvo siempre la misma respuesta pues todos los participantes salian de casa al haber sido realizado el cuestionario en la farmacia.

La mayoría de los entrevistados fueron mujeres $(60,5 \%)$, siendo casi la mitad diabéticos (42,8\%). La muestra obtenida representa la población que acude a la farmacia, aunque se aproxima a la población general ya que según el Instituto Nacional de Estadística, el sexo predominante en la vejez es el femenino, suponiendo un $63,8 \%$ de la población general [26].

Los resultados obtenidos en el presente estudio ponen de manifiesto que el 7,1\% de las personas mayores de 65 años que acuden a las farmacias españolas presentan malnutrición, coincidiendo con la prevalencia que existe a nivel nacional (5-8\%) [8]. Malnutrición que resultó mayor en mujeres que en los hombres. Resultó además que el 33,8\% de los entrevistados presentaban riesgo de malnutrición, por lo que en conjunto el porcentaje de mayores de 65 años con malnutrición y riesgo de padecerla que detectamos es del 40,9\%.

Existe una gran variabilidad de resultados al comparar los obtenidos en este estudio con otros diferentes realizados en España con personas mayores no institucionalizadas. A excepción del realizado en 2009 por De la Montaña et al [27], que obtienen un porcentaje de pacientes con malnutrición del 12,5\% y riesgo de desnutrición del 57,5\%, los resultados obtenidos en los demás estudios revisados son inferiores a los del nuestro: 0,5\% a 3,8\% en malnutrición y 4,5\%-30,3 en riesgo de malnutrición [15,28-33]. Por lo que los datos obtenidos en nuestro estudio representan el porcentaje de prevalencia de malnutrición y de riesgo de malnutrición más elevados en la actualidad de España en ancianos no institucionalizados.

En el ámbito europeo, con los mismos parámetros de medición, nos encontramos con porcentajes de malnutrición ligeramente inferiores al nuestro (1-3\%) [34-36], pero con una población en riesgo superior (48\%) [34].
Vivir solo es un factor de riesgo importante de malnutrición [26]. En la respuesta a la pregunta $\mathrm{G}(¿ E \mathrm{El}$ paciente vive independiente en su domicilio?) resulta que el $82 \%$ de las personas encuestadas en nuestro estudio viven solas, por lo que ésta puede ser una razón del elevado porcentaje de malnutrición o riesgo de malnutrición encontrado.

$\mathrm{Al}$ analizar los resultados del cuestionario MNA por tramos de edad, vemos que a mayor edad, la puntuación del MNA es menor y que la diferencia entre los sexos no existe para el tramo de edad entre 65 y 74 años; pero si hemos encontrado que la puntuación es mayor en los hombres que en las mujeres de 75 a 84 años, y menor en hombres de 85 o más años. Estos resultados no se pueden comparar con otros estudios ya que no establecen los mismos tramos de edad, pero también aprecian una tendencia al incremento del riesgo que coincide con el proceso natural de envejecimiento que condiciona la capacidad nutricional de las personas [29].

La media del IMC fue $28,6 \mathrm{~kg} / \mathrm{m}^{2}$ (sobrepeso), dato similar al encontrado por Méndez et al [37] en 2013 y un poco mayor que Lardies et al [11] en 2015. En nuestro estudio más de las tres cuartas partes de los participantes tenían sobrepeso/obesidad y solamente $7(0,7 \%)$ presentaban desnutrición según este parámetro antropométrico (SENPE-SEGG) [26]. Este 1\% de desnutrición obtenido mediante el IMC está muy lejos del 7,1\% resultante del cuestionario MNA. La razón es que con el cuestionario no medimos desnutrición sino malnutrición, que engloba valores por debajo y por encima del IMC, ya que personas con IMC en el rango de sobrepeso/obesidad, incluso en normalidad, pueden padecer malnutrición. Así, con el IMC el porcentaje de desnutrición entre sexos no varía, pero sí es mayor el porcentaje de sobrepeso/ obesidad en hombres que en mujeres $\mathrm{y}$, sin embargo, según el MNA el riesgo de desnutrición y malnutrición es mayor en las mujeres.

Dentro de las medidas antropométricas, la CP es un indicador del tejido muscular y graso, parámetro de gran importancia en la determinación de la composición corporal en personas mayores, aunque todavía no está validado. Actualmente no existe ningún punto de corte internacionalmente 
aceptado y utilizado para clasificar la $\mathrm{CP}$, por lo que estudios previos $[21,38]$ usaron como punto de corte el que figura en el cuestionario MNA.

La puntuación del MNA de los participantes de nuestro estudio con $\mathrm{CP}<31 \mathrm{~cm}(19,6 \pm 5,6)$ es significativamente $(\mathrm{p}<0.001)$ inferior a la puntuación del MNA de los encuestados con $\mathrm{CP}>31 \mathrm{~cm}(24,6 \pm 3,3)$. Estos valores concuerdan con la asociación que hicieron Cuervo et al [21], donde para establecer la relación entre una situación de malnutrición y la $\mathrm{CP}$ seleccionaron al grupo con $\mathrm{MNA} \leq 23,5$ puntos como riesgo de malnutrición y aquellos con $>24$ puntos como población sin riesgo. Encontraron que la media de los individuos con menor $\mathrm{CP}$ estaba dentro del intervalo definido como riesgo de desnutrición y la media de aquellos con mayor $\mathrm{CP}$ indicaba no existir riesgo de desnutrición, tanto en hombre como mujeres.

Con respecto a las diferencias entre diabéticos y no diabéticos, fue difícil comparar nuestros resultados con otros estudios de carácter similar. En 2015, Lardiés et al [11] compararon el estado nutricional entre ambos grupos en personas institucionalizadas. Obtuvieron una proporción menor de diabéticos $(21,7 \%)$ en comparación con la nuestra $(42,8 \%)$, con un predominio de las mujeres $(57,6 \%)$ similar al nuestro (60,5\%). Encontraron diferencias estadisticamente significativas $(p<0,05)$ en el peso $(65,3 \pm 13,2)$, IMC $(27,9 \pm 5,4)$ y otras medidas antropométricas que en nuestro estudio no se realizaron, a diferencia de nuestro trabajo donde la única diferencia estadísticamente significativa fue el IMC, mayor en diabéticos $(29,0 \pm 4.8)$. En cuanto a prevalencia de malnutrición encuentran mayor riesgo (45,5\% muestra total y 52,2\% diabéticos), coincidente con el hecho de que el riesgo de malnutrición actual en España es mayor en personas mayores institucionalizadas que en no institucionalizadas.

Desde principios del siglo $\mathrm{xx}$ se ha producido en España un significativo crecimiento de la población debido a la mejora de las condiciones de vida, la alimentación, la higiene y la atención sanitaria. Este crecimiento ha sido paralelo a un proceso de envejecimiento como consecuencia de la baja tasa de natalidad y del aumento de la longevidad [25], llegando hasta los 82,1 años de esperanza de vida media en la actualidad. En esta población mayor se observa un aumento en el riesgo de malnutrición que puede provocar el desarrollo y/o empeoramiento de ciertas patologías [39].

Los resultados de nuestro estudio muestran que la malnutrición y el riesgo de malnutrición son un problema importante en la mayoría de las personas con más de 65 años afectando en mayor grado a mujeres y a diabéticos. Es importante, por tanto, realizar estudios para conocer y prevenir estas situaciones de malnutrición en las personas mayores, desarrollando programas educacionales y asistenciales que contribuyan a mejorar su estado nutricional y por tanto su calidad de vida. El estudio que se presenta muestra que la farmacia comunitaria es un ámbito adecuado para la detección de este tipo de problemas y por lo tanto puede colaborar de manera significativa en su solución.

\section{Agradecimientos}

A todos los farmacéuticos participantes en el estudio: Mera Gallego I, Ferrer Ubeda JA, Moya Aragón C, Ruiz Lara AM, Cortés Botella P, Mera Gallego R, Fornaris Ramis A, Daries Alfonso C, Calle Pérez JL, Hernández Marrero MR, Luque del Moral R, Castillo Fernández AR, Escribá Martí $\mathrm{P}$, Fernández Veiga A, García Zaragoza ME, Granell Vidal L, Molinero Crespo A, Muruzábal Ardanaz C, Pérez Belda E, Rodríguez Sampedro A, Soler Saura JL, Adzet Panades E, Alba Palomo JR, Amat Olcina C, Fondevila Estévez S, García Rodríguez P, Martí Escursell MR, Martínez-Moya Bernal P, Montalbán Soler L, Morillo Lisa RM, Plaza Zamora FJ, Ruiz de Castañeda Gallego J, Salar Ibáñez L, Sánchez Marcos N, Santamaría López JM, Velert Vila J, Alonso Lovera P, Álvarez Cejudo C, Baltar Bescansa 0, Barrionuevo Torres A, Burgos Reques A, Cantero Angulo JJ, de Andrés Dirube A, de Diego Colilla V, Esteve González A, Ferrer Estrela F, García Llaneza E, García Moreno L, García Vivancos MC, Martín Lázaro R, Méndez Mora-Figueroa P, Moreno Cascales MC, Murillo Fernández $\mathrm{MD}$, Orenes Barceló $\mathrm{M}$, Pascual Rovira I, Peris Molina MT, Raíces Madriñán MC, Ruiz Martínez I, San Martín Ursa R, Tejedor García N, Villarroya Pérez A, Peña Ros JE, Urcullu Donat B, Souto Bethencourt I, Pérez y Piedrahita MN, Sánchez Martínez RC,
Carbonell Ferrer L, Gómez Pérez M, Martínez Márquez M, Carrasco Fernández G, Marrón Souto E, Fornos Pérez JA.

\section{Referencias bibliográficas}

1. Salvá Casanovas A, Serra Rexach JA. Pérdida de peso y desnutrición en las personas mayores: epidemiología. En: Planas M [coordinadora]. Valoración nutricional en el anciano. Documento de consenso. Sociedad Española de Nutrición Parenteral y Enteral y Sociedad Española de Geriatría y Gerontología. 2007. Galénitas-NigraTrea. Págs. 15-40. [Acceso 12/1/2017]. Disponible en: http://www.senpe.com/ documentacion/consenso/senpe_valoracion_nutricional_anciano.pdf

2. Organización de las Naciones Unidas. World Population Ageing, 1950-2050. Department of Economic and Social Affairs. 2007. [Acceso 14/12/2016] Disponible en: http://www.un.org/ esa/population/publications/worldageing 19502050/

3. Sastre A. Envejecimiento: cambios y consecuencias. Nutrición. En: Serrano M, Cervera P, López C, Ribera JM, Sastre A. Guía de alimentación para personas mayores. Madrid: Ergón; 2010. Págs. 13-25. [Acceso 11/1/2017]. Disponible en: http://www.institutodanone.es/assets/guia_de_alimentacion_para_personas_mayores.pdf

4. García de Lorenzo M, García Luna P, Marsé P. El libro blanco de la desnutrición clínica en España. Madrid: Iberoaméricana; 2010.

5. Montejano Lozoya R, Ferrer Diego RM, Clemente Marín G, Martínez Alzamora Nieves. Estudio del riesgo nutricional en adultos mayores autónomos no institucionalizados. Nutr Hosp 2013; 28(5):1490-1498.

6. García de Lorenzo A, Álvarez J, Calvo MV, Ulíbarri I, Del Río J, Galbán C, et al. Conclusiones del II Foro de Debate SENPE sobre desnutrición hospitalaria. Nutr Hosp 2005; 20(2):82-87.

7. Ministerio de Sanidad y Consumo [Internet]. Clasificación Internacional de Enfermedades. 9a Revisión Modificación Clínica. 9a ed. 2014, Madrid. [Acceso 16/1/2017]. Disponible en: www. msssi.gob.es/estadEstudios/estadisticas/ docs/CIE9MC_2014_def_accesible.pdf

8. Milá Villarroel R, Formiga F, Duran Alert P, Abellana Sangrà R. Prevalencia de malnutrición en la población anciana española: Una revisión sistemática. Med Clin (Barc) 2012; 139(11):502-508. doi:10.1016/j.medcli.2012.04.008

9. Rentero Redondo L, Iniesta Navalón C, Gascón Cánovas JJ, Tomás Jiménez C, Sánchez Álvarez C. Desnutrición en el 
paciente anciano al ingreso hospitalario, un viejo problema sin solucionar. Nutr Hosp. 2015; 32(5):2169-2177.

10. Fernández López MT, Fidalgo Baamil 0, López Doldán C, Bardasco Alonso ML, De Sas Prada MT, Lagoa Labrado F et al. Prevalencia de desnutrición en pacientes hospitalizados no críticos. Nutr Hosp 2015; 31(6): 2676-2684.

11. Lardiés Sánchez B, López Valverde ME, Pérez Fernández L, Albero Gamboa R, Sanz Paris A, Soriguer F. Prevalencia de diabetes mellitus en una población de ancianos institucionalizados y comparación de antropometría, composición corporal y estado nutricional entre diabéticos y no diabéticos. Av Diabetol. 2015; 31(Espec Congr):105.

12. Rasheed S, Woods RT. Malnutrition and quality of life in older people: a systematic review and meta-analysis. Ageing Res Rev. 2012; S:1568-1637.

13. García A, Alvarez J, De Man F. Envejecimiento y desnutrición; un reto para la sostenibilidad del SNS conclusiones del IX Foro de Debate Abbott-SENPE. Nutr Hosp. 2012; 27(4):1060-1064.

14. Vega Piñeiro B. Aspectos diferenciales de la nutrición en los pacientes ancianos con diabetes. Av Diabetol. 2010; 26:307-313. doi:10.1016/S11343230(10)65002-8

15. Consejo General de Colegios Oficiales de Farmacéuticos [Internet]. Vocalía Nacional de Alimentación. Plan de educación nutricional por el farmacéutico (Plenufar III): Educación nutricional a las personas mayores. Madrid: CGCOF; 2006. Disponible en: http://envejecimiento.csic.es/documentos/documentos/plenufar-resultados-01.pdf

16. Baixauli V, Vanrell B, Llull R, González A, Servera M. Estudio de detección de desnutrición en farmacia comunitaria en personas mayores de 65 años (proyecto D’NUT). Farmacéuticos Comunitarios [Internet] 2014; 6(Supl 1). [Acceso 4/2/2017]. Disponible en: http://www.farmaceuticoscomunitarios.org/es/journal-article/estudio-deteccion-desnutricion-farmacia-comunitaria-personas-mayores-65-anos

17. Muñoz P, Manchón MG. Valoración nutricional de mayores. Seguimiento desde la farmacia comunitaria. Farmacéuticos Comunitarios [Internet] 2014; 6(Supl 1). [Acceso 4/2/2017]. Disponible en: http://www.farmaceuticoscomunitarios.org/es/journal-article/ valoracion-nutricional-mayores-seguimiento-desde-farmacia-comunitaria

18. Mera I, Fornos JA, Ferrer JA, Ruiz A, Mera R, García P, et al. Evaluación del estado nutricional en personas mayores de 65 años con diabetes en farmacia comunitaria. Farmacéuticos comuni- tarios [Internet] 2016; 8 (Supl 1). [Acceso 4/2/2017]. Disponible en: http:// www.farmaceuticoscomunitarios.org/ es/journal-article/evaluacion-del-estado-nutricional-personas-mayores-65-anos-con-diabetes-farmacias

19. Llull R, Labat MP, Baixauli V, Moranta F, Huarte J. Proyecto "El farmacéutico que necesitas": servicio de detección de desnutrición en pacientes ancianos. Farmacéuticos Comunitarios [Internet] 2016; 8(Supl 1). [Acceso 5/2/2017] Disponible en: http:// farmaceuticoscomunitarios.org/es/ journal-article/proyecto-farmaceutico-que-necesitas-servicio-deteccion-desnutricion-pacientes

20. Casimiro C, García de Lorenzo A, Usán L, Grupo de Estudio Cooperativo Geriátrico. Evaluación del riesgo nutricional en pacientes ancianos ambulatorios. Nutr. Hosp. 2001 ; 16(3):97-103

21. Cuervo M, García A, Ansorena D, Sánchez A, Martínez M, Astiasarán I, et al. Nutritional assessment interpretation on Spanish community-dwelling elders through the Mini Nutritional Assessment test. Public Health Nutr. 2009 Jan; 12(1):82-90. doi:10.1017| S136898000800195X

22. Instituto Nacional de Estadística. Proyección de la Población de España a Largo Plazo (2009-2049). Madrid [Internet] [Acceso 16/1/2017]. Disponible en: http://www.ine.es/metodologia/t20/t2030251.pdf

23. Soriguer F, Goday A, Bosch-Comas A, Bordiú E, Calle-Pascual A, Carmena R, et al. Prevalence of diabetes mellitus and impaired glucose regulation in Spain: the Di@bet.es Study. Diabetologia 2012; 55(1):88-93. doi:10.1007/ s00125-011-2336-9

24. Nestlé Nutrition Institute [Internet]. MNA $^{\circledR}$ Mini Nutritional Assessment. [Acceso 18/1/2017]. Disponible en: www.mna-elderly.com/default.html

25. Planas M [coordinadora] [Internet]. Valoración nutricional en el anciano. Recomendaciones prácticas de los expertos en geriatría y nutrición. Documento de consenso. Sociedad Española de Nutrición Parenteral y Enteral y Sociedad Española de Geriatría y Gerontología. 2007. Galénitas-NigraTrea. Págs. 15-40. [Acceso 12/1/2017]. Disponible en: http://www.senpe.com/ documentacion/consenso/senpe_valoracion_nutricional_anciano.pdf

26. Abellán García A, Pujol Rodríguez R [Internet]. Un perfil de las personas mayores en España, 2015. Indicadores estadísticos básicos. Informes envejecimiento en red. [Acceso 16/1/2017]. Disponible en: envejecimiento.csic.es/ documentos/documentos/enred-indicadoresbasicos15.pdf.

27. De la Montaña Miguélez J, Areal Salve C, Míguez Bernárdez M. Evaluación del riesgo nutricional mediante el MNA en una población anciana no institucionalizada. Archivo Latinoamericanos de Nutrición 2009; 59(4):390-395.

28. Salvà A, Jose Bleda M, Bolibar I. The Mini Nutritional Assessment in clinical practice. Nestle Nutr Workshop Ser Clin Performan Programme 1999; 1:123-129. doi:10.1159/000062960

29. Ramón JM, Subirà C. Prevalencia de malnutrición en la población anciana española. Med Clin 2001; 217:766-770. doi:10.1016/S0025-7753(01)72252-X

30. Tur JA, Colomer M, Monino M, Bonnin T, Llompart I, Pons A. Dietary intake and nutritional risk among free-living elderly people in Palma de Mallorca. J Nutr Health Aging. 2005; 9:390-396.

31. Morillas J, García Talavera N, Martín Pozuelo G, Reina AB, Zafrilla P. Detección del riesgo de desnutrición en ancianos no institucionalizados. Nutr Hosp. 2006; 21(6):650-656

32. Salvà $\mathrm{A}$, Lucas R. Qualitat de vida a les persones grans a Catalunya. Barcelona: Fundació Viure I Conviure; 2007

33. Jiménez Sanz M, Sola Villafranca JM, Pérez Ruiz C, Turienzo Llata MJ, Larrañaga Lavin F, Mancebo Satamaría MA et al. Estudio del estado nutricional de los ancianos de Cantabria. Nutr Hosp. 2011: 26(2):345-354.

34. Soini H, Routasalo P, Lagstrom H. Characteristics of the Mini Nutritional Assessment in elderly home-care patients. Eur J Clin Nutr. 2004; 58:6470. doi:10.1038/sj.ejcn. 1601748

35. De Groot LC, Beck AM, Schroll M, Van Staveren WA. Evaluating the DETERMINE Your Nutritional Health Checklist and the Mini Nutritional Assessment as tools to identify nutritional problems in elderly Europeans. Eur J Clin Nutr. 1998; 52(12):877-83. doi:10.1038/sj.ejcn.1600658

36. Guigoz Y. The Mini Nutritional Assessment (MNA) review of the literature. What does it tell us? J Nutr Health Aging. 2006: 10(6):466-485.

37. Méndez Estévez E, Romero Pita J, Fernández Domínguez MJ, Troitiño Álvarez P, García Dopazo S, Jardón Blanco $\mathrm{M}$ et al. ¿Tienen nuestros ancianos un adecuado estado nutricional? ¿Influye su institucionalización? Nutr Hosp. 2013; 28(3):903-913.

38. Rolland Y, Lauwers-Cances V, Cournot M, Nourhashémi F, Reynish W, Rivière D et al. Sarcopenia, calf circumference, and physical function of elderly women: a croos-sectional study. J Am Geriatr Soc. 2003;51(8):1120-1124. doi:10.1046/j.1532-5415.2003.51362.x

39. Saka B, Kaya O, Ozturk GB, Erten N, Karan MA. Malnutrition in the elderly and its relationship with other geriatric syndromes. Clinical Nutrition 2010; 29:745-748. doi:10.1016/j. clnu.2010.04.006 


\section{Anexos}

Anexo 1. Cuestionario Mini Nutritional Assessment (MNA)

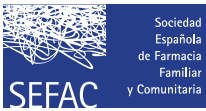

\section{Evaluación del estado nutricional Mini Nutritional Assessment MNA ${ }^{\mathrm{TM}}$}

\begin{tabular}{|c|c|c|c|c|c|c|c|}
\hline NOMBRE & & & IDOS & & & & FECHA \\
\hline$\overline{S E X O}$ & $\overline{E D A D}$ & $\overline{\mathrm{PESO}(\mathrm{kg})}$ & $\overline{\text { TALLA (cm) }}$ & $\begin{array}{l}\text { ALTURA TALÓN-RODILLA } \\
\text { (en caso de movilidad reducida) }\end{array}$ & $\mathrm{IMC}$ & ¿DIABÉTICO? & FECHA DE DIAGNÓSTICO \\
\hline
\end{tabular}

\section{Cribaje}

A ¿Ha perdido el apetito? ¿Ha comido menos por falta de apetito, problemas digestivos, dificultades de masticación 0 deglución en los últimos 3 meses?

$0=$ anorexia grave

1 = anorexia moderada

$2=\sin$ anorexia

B Pérdida reciente de peso $(<3$ meses $)$

$0=$ pérdida de peso $>3 \mathrm{~kg}$

$1=$ no lo sabe

2 = pérdida de peso entre 1 y $3 \mathrm{~kg}$

3 = no ha habido pérdida de peso

C Movilidad

0 = de la cama al sillón

1 = autonomía en el interior

2 = sale del domicilio

D ¿Ha tenido una enfermedad aguda o situación de estrés psicológico en los últimos 3 meses? $0=$ sí $2=$ no

E Problemas neuropsicológicos

$0=$ demencia 0 depresión grave

1 = demencia 0 depresión moderada

$2=$ sin problemas psicológicos

F Índice de masa corporal $\left(\mathrm{IMC}=\right.$ peso $/(\text { talla })^{2}$ en $\left.\mathrm{kg} / \mathrm{m}^{2}\right)$

$0=I M C<19$

$1=19 \leq \mathrm{IMC}<21$

$2=21 \leq \mathrm{IMC}<23$

$3=I M C \geq 23$

Evaluación del cribaje (subtotal máx. 14 puntos)

12 puntos o más normal

11 puntos o menos posible malnutrición

\section{Evaluación}

G ¿El paciente vive independiente en su domicilio?

$0=$ no $1=$ sí

H ¿Toma más de 3 medicamentos al día? $0=$ sí $1=$ no

I ¿Úlceras o lesiones cutáneas?

0 = sí $1=$ no

J ¿Cuántas comidas completas toma al día?

(Equivalentes a dos platos y postre)

$0=1$ comida

$1=2$ comidas

- 3 comidas

$\mathrm{K}$ ¿Consume el paciente

- productos lácteos al menos una vez al día?

- huevos o legumbres

102 veces a la semana?

- carne, pescado 0 aves, diariamente?

$0,0=001$ síes

$0,5=2$ síes

$1,0=3$ síes

sí $\square$ no $\square$

sí $\square \quad$ no $\square$

sí $\square$ no $\square$

¿Consume frutas 0 verduras al menos 2 veces al día? $0=$ no $1=$ sí

M ¿Cuántos vasos de agua u otros líquidos toma al día? (agua, zumo, café, té, leche, vino, cerveza...)

$0,0=$ menos de 3 vasos

$0,5=$ de 3 a 5 vasos

$1,0=$ más de 5 vasos

$\mathrm{N}$ Forma de alimentarse

$0=$ necesita ayuda

1 = se alimenta solo con dificultad

2 = se alimenta solo sin dificultad

0 ¿Se considera el paciente que está bien nutrido? (problemas nutricionales)

$0=$ malnutrición grave

1 = no lo sabe 0 malnutrición moderada

$2=$ sin problemas de nutrición

P En comparación con las personas de su edad,

¿cómo encuentra el paciente su estado de salud?

$0,0=$ peor

$0,5=$ no lo sabe

$1,0=$ igual

$2,0=$ mejor

Q Circunferencia braquial ( $\mathrm{CB}$ en $\mathrm{cm}$ )

$0,0=\mathrm{CB}<21$

$0,5=21 \leq \mathrm{CB} \leq 22$

$1,0=\mathrm{CB}>22$

$\mathrm{R}$ Circunferencia de la pantorrilla (CP en $\mathrm{cm}$ )

$0=\mathrm{CP}<31 \quad 1=\mathrm{CP} \geq 31$

Evaluación (máx. 16 puntos)

Cribaje

Evaluación global (máx. 30 puntos)

Evaluación del estado nutricional

De 17 a 23,5 puntos

riesgo de malnutrición

Menos de 17 puntos

malnutrición 


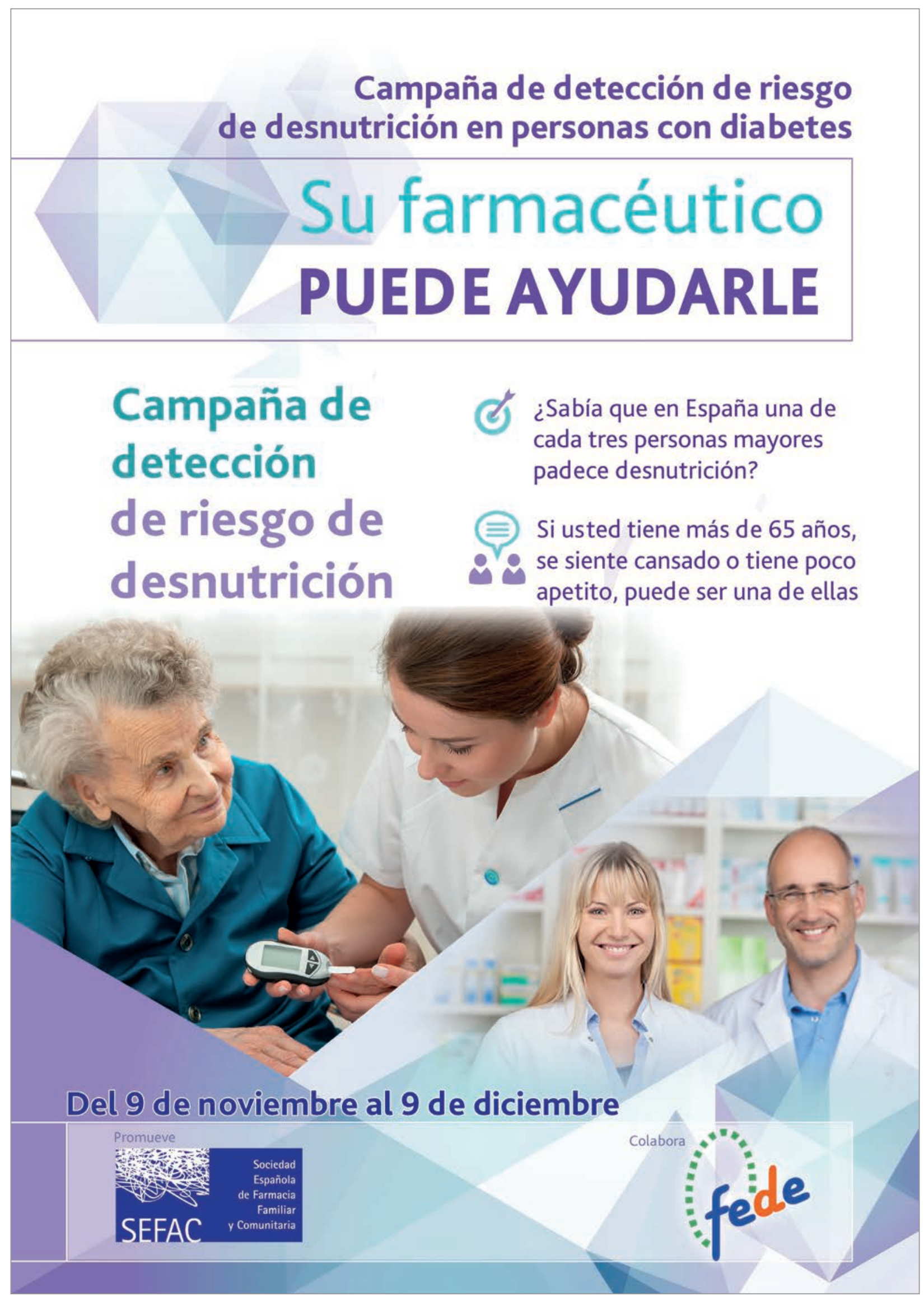




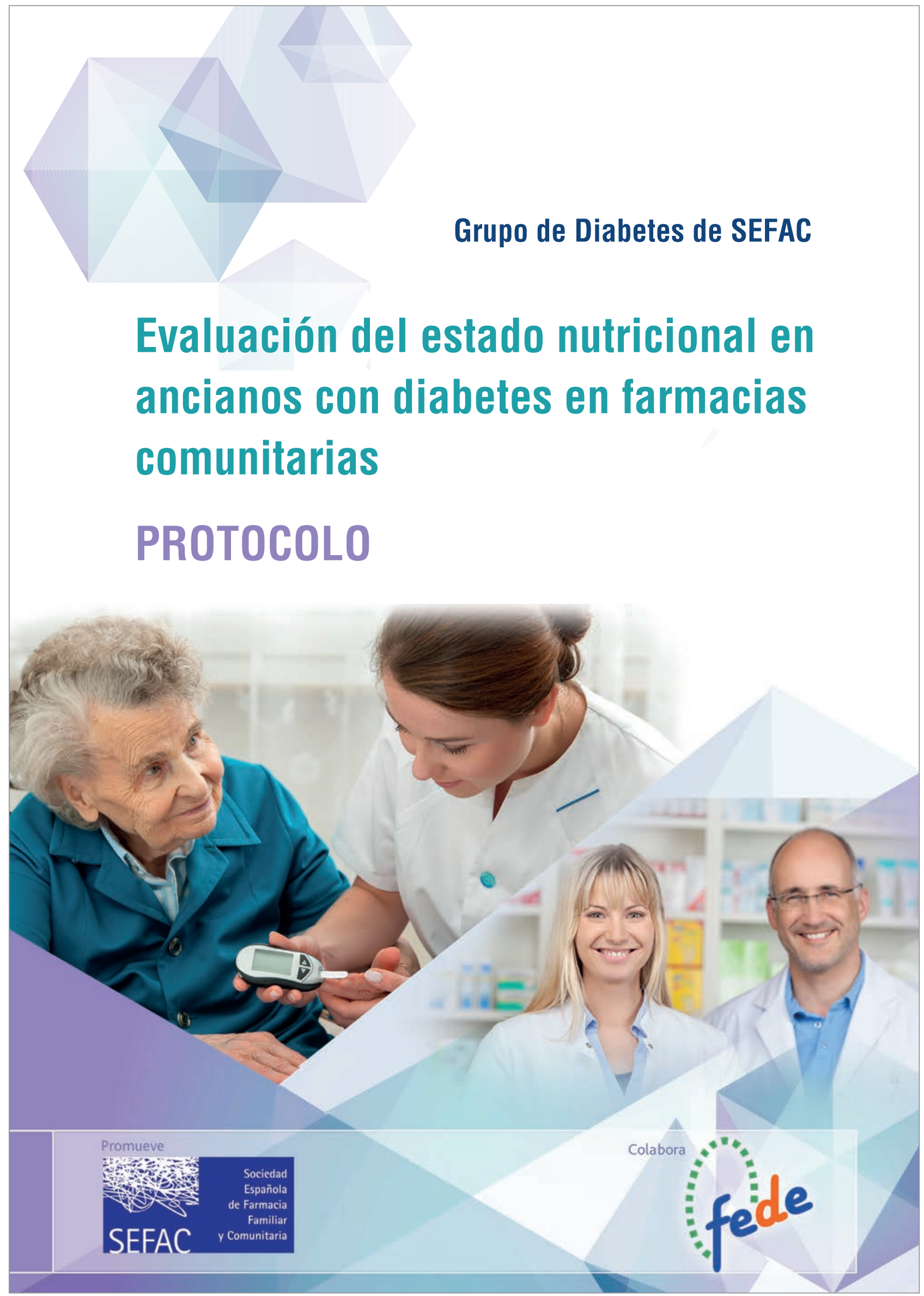


Hay una gran variabilidad en la prevalencia de pérdida de peso en las personas mayores. Los estudios epidemiológicos han mostrado que la mayoría de las personas mayores mantienen su peso dentro de los límites de la normalidad. Sin embargo, entre un 15 y un $20 \%$ experimenta una pérdida de peso definida como una pérdida del $5 \%$ de su peso usual. Este porcentaje alcanza el $27 \%$ en poblaciones seleccionadas de alto riesgo, como las que reciben servicios a domicilio'.

Muchos factores se han asociado a una mayor prevalencia de pérdida de peso. Entre ellos destacamos: edad avanzada, discapacidad, comorbilidad, deterioro cognitivo, y también con factores sociales como un nivel educativo bajo o la viudedad. La incidencia de pérdida no voluntaria de peso varía entre un 1,3 y un $8 \%$ dependiendo del lugar del estudio ${ }^{1}$.

En las personas ancianas con diabetes, desde el punto de vista fisiopatológico, con el envejecimiento se produce de manera fisiológica una pérdida de sensibilidad a la insulina. El sobrepeso y la obesidad son frecuentes en la población anciana con diabetes; además, con el envejecimiento se produce una redistribución de la grasa corporal, con un incremento de la adiposidad visceral. Otros factores que contribuyen a la resistencia insulínica son las modificaciones en el estilo de vida que se dan en individuos de edad avanzada (reducción de la actividad física, cambios en la dieta con una mayor ingesta de alimentos ricos en grasas saturadas, ricas en azúcares simples y pobres en carbohidratos complejos) y un mayor consumo de fármacos que interfieren el metabolismo hidrocarbonado. Teniendo en cuenta esto, Ios ancianos con diabetes son pacientes con alto riesgo de desnutrición, llegando a padecerla, según algunos estudios, el $50 \%$ de los mismos².

Es nuestra intención comprobar si existe diferencia en el estado nutricional del anciano, según sea diabético o no.

\section{OBJETIVO}

Evaluar el estado nutricional de las personas de más de 65 años con diabetes, que acuden a la farmacia comunitaria y compararla con personas no diabéticas.

\section{METODOLOGÍA}

Estudio observacional transversal llevado a cabo en las farmacias comunitarias de SEFAC, desde el 9 de noviembre hasta el 9 de diciembre de 2015.

\section{Criterios de inclusión}

Personas de 65 años 0 más, usuarios que acuden a la farmacia, con autonomía suficiente para contestar y que consienten hacerlo.

\section{Variables a medir}

Edad (años), sexo (H/M), peso $(\mathrm{kg})$, talla $(\mathrm{m})$, IMC $\left(\mathrm{kg} / \mathrm{m}^{2}\right)$, altura suelo-rodilla (sentados) (cm), circunferencia braquial $(\mathrm{cm})$, circunferencia de la pantorrilla (cm), diabetes (Sí/No), fecha de diagnóstico de diabetes (si procede), enfermedades (tipo), medicamentos (tipo) y las preguntas del Mini Nutritional Assessment (MNA) ${ }^{3}$ (puntos).

\section{PROCEDIMIENTO $1^{\text {a }}$ FASE}

1. Ofrecimiento a los usuarios de 650 más años, que acuden a la farmacia entre el 9 de noviembre y 9 de diciembre de 2015.

2. Realizar las determinaciones antropométricas según la guía ${ }^{4}$ adjunta: peso, talla, circunferencia braquial y circunferencia de la pantorrilla. Cálculo del IMC $\left(\mathrm{kg} / \mathrm{m}^{2}\right)$ (con el peso en $\mathrm{kg}$ y la talla en $\mathrm{m}$ y al cuadrado).

En el caso de que la persona tenga problemas de movimiento, dificultad para ponerse de pie, o columna deformada, se medirá la altura talón-rodilla (desde el suelo hasta la parte superior de la rodilla $)^{5,6}$.

3. Cumplimentación de la encuesta: datos (edad, sexo, etc...), diabetes (Sí/No) y el MNA (las dos partes del mismo y con su puntuación).

\section{Intervención del farmacéutico}

a. Estado nutricional normal (24-30 puntos): informar al paciente de que no presenta riesgo de desnutrición y recordarle, entregándole información escrita, la importancia de los hábitos higiénico-dietéticos saludables.

b. Riesgo de desnutrición (17-23,5 puntos): explicar qué significa. Informarle, entregándole información escrita, de la importancia de los hábitos higiénico-dietéticos saludables. Y a criterio del farmacéutico, llevar a cabo una intervención y seguimiento (anexo 2) o derivación al médico.

c. Desnutrición (<17 puntos): explicarle lo que significa. Informarle, entregándole información escrita, de la importancia de los hábitos higiénico-dietéticos saludables. Y derivarle al médico con una copia del MNA.

4. Introducir los datos ANÓNIMOS en la página web de SEFAC, www.sefac.org, accediendo con las claves de socio y pulsando en el banner del estudio, hasta el 16 de diciembre.

Al término de este proyecto, para aquellos farmacéuticos que quieran continuar con intervenciones para mejorar el estado nutricional detectado, pasaríamos a la segunda fase (Anexo 2). 
Anexo 4. PNT para el cálculo del IMC

\begin{tabular}{|c|c|c|}
\hline Farmacia & $\begin{array}{c}\text { PROCEDIMIENTO DE DETERMINACIÓN DEL } \\
\text { ÍNDICE DE MASA CORPORAL (IMC) }\end{array}$ & $\begin{array}{c}\text { PNT: 01 } \\
\text { Edición: 00 } \\
\text { Fecha:20/07/2015 }\end{array}$ \\
\hline Redactado por & Revisado por & Aprobado por \\
\hline
\end{tabular}

\section{Objeto y aplicación}

Definir las operaciones y pautas a seguir para la determinación del peso corporal y estatura con la finalidad de calcular el IMC en la farmacia comunitaria (FC).

Este procedimiento se aplicará a aquellos usuarios que se incluyan en el programa de detección del riesgo de malnutrición.

\section{Definiciones}

Índice de masa corporal: relación entre el peso en $\mathrm{kg}$ y la estatura en metros elevada al cuadrado $\left(\mathrm{kg} / \mathrm{m}^{2}\right)$ que se utiliza como método de diagnóstico del sobrepeso y de la obesidad o adiposidad corporal.

\begin{tabular}{|l|l|}
\hline IMC $\left(\mathrm{kg} / \mathrm{m}^{2}\right)$ & Categoría \\
\hline$<18$ & Desnutrición \\
\hline 18 a 24 & Normal \\
\hline 25 a 29 & Sobrepeso \\
\hline 30 a 39 & Obesidad \\
\hline$\geq 40$ & Obesidad mórbida \\
\hline
\end{tabular}

\section{Descripción}

Equipo y material

- Báscula pesa personas

- Tallimetro

- Hoja de registro

\section{Consideraciones generales sobre el equipo y material}

- Las básculas pueden ser:

- Mecánicas que a su vez pueden ser de columna con pesas deslizantes, denominadas romanas, o de columna con disco giratorio.

- Electrónicas.

- Mantener las básculas sobre una superficie horizontal no expuesta a vibraciones.

- Las básculas tienen que estar homologadas según la Directiva Europea 90/384/CEE.

- Los tallímetros pueden ser mecánicos o electrónicos que pueden estar incorporados en la báscula.

\section{Operación de medida del peso corporal}

- Para medir el peso situar a la persona en el centro de la plataforma de la báscula, asegurando que distribuye el peso por igual en ambas piernas, sin que el cuerpo contacte con nada de su alrededor y con los brazos colgando a lo largo del cuerpo.

- La persona debe llevar ropa ligera y se realizará el pesaje sin los zapatos.

- Se puede realizar el pesaje dos veces y calcular el promedio para minimizar los posibles errores.

- Registrar el resultado en la hoja de recogida de datos en base al kilogramo más cercano.

\section{Operación de medida de la estatura}

- Comprobar que la superficie del suelo es lisa y firme.

- Pedir al paciente que se quite los zapatos y se coloque derecho con los talones juntos y con los glúteos y hombros pegados al tallímetro o estadiómetro.

- Los brazos deben colgar relajados con las palmas contra los muslos. 
- Tomar la medida con el paciente bien erguido, con la mirada al frente y la cabeza derecha, no inclinada hacia atrás.

- Asegurarse de que los talones estén pegados al suelo.

- Descender el medidor del estadiómetro hasta que toque la parte superior de la cabeza.

- Registrar el resultado de la medida redondeando al centímetro más cercano

- Los tallímetros electrónicos darán la medida automáticamente.

Cálculo del Índice de Masa Corporal (IMC)

El Índice de Masa Corporal es el resultado de dividir el peso en kg por el cuadrado de la talla en metros:

$$
\text { IMC }=\text { Peso }(\mathrm{kg}) / \text { Talla }(\mathrm{m})^{2}
$$

Registrar el resultado en la hoja de recogida de datos.

Menos de $19 \mathrm{~kg} / \mathrm{m}^{2}$................. 0 puntos

$19-21 \mathrm{~kg} / \mathrm{m}^{2}$........................... 1 puntos

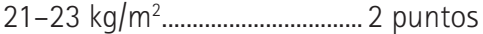

Mayor de $23 \mathrm{~kg} / \mathrm{m}^{2} \ldots \ldots \ldots \ldots \ldots \ldots . . . . .3$ puntos

\section{Control de cambios}

\begin{tabular}{|l|l|l|}
\hline Edición & Cambio realizado & Fecha \\
\hline & & \\
\hline & & \\
\hline & & \\
\hline
\end{tabular}


Anexo 5. PNT para el cálculo de la circunferencia braquial y de la pantorrilla

\begin{tabular}{|c|c|c|}
\hline Farmacia & $\begin{array}{c}\text { PPROCEDIMIENTO DE DETERMINACIÓN } \\
\text { DE LA CIRCUNFERENCIA BRANOUIAL Y } \\
\text { PANTORRILLA }\end{array}$ & $\begin{array}{c}\text { PNT: } 01 \\
\text { Edición: } 00 \\
\text { Fecha:20/07/2015 }\end{array}$ \\
\hline Redactado por & Revisado por & Aprobado por \\
\hline
\end{tabular}

\section{Objeto y aplicación}

Definir las operaciones y pautas a seguir para la determinar la circunferencia braquial (CB) y de la pantorrilla (CP).

Este procedimiento se aplicará a aquellos usuarios que se incluyan en el programa detección del riesgo de desnutrición.

\section{Definiciones}

El PB es un indicador de la pérdida de masa muscular del brazo que se basa en la medida de la circunferencia del brazo en el punto medio situado entre el extremo del acromion de la escápula y el olecranon del cúbito.

El PP es un indicador del tejido muscular y graso de gran importancia en la determinación de la composición corporal en personas mayores que se basa en la medición de la máxima circunferencia de la pierna por debajo de la articulación de la rodilla.

\section{Descripción}

\section{Equipo y material}

- Cinta métrica no extensible

- Hoja de registro

\section{Determinación circunferencia branquial}

- Ubique a la persona en supinación o decúbito dorsal, con el antebrazo flexionado por encima del cuerpo, formando un ángulo de 90 grados y con la palma hacia abajo. El brazo debe quedar paralelo al tronco.

- Palpe la esquina superior de la escapula hasta localizar el vértice del acromio.

- Mida con una cinta métrica desde este punto anatómico hasta el borde inferior del olecranon, por debajo del codo.

- Calcule el punto medio y márquelo, usando para ello el lapicero.

- Extienda el brazo del sujeto a lo largo del cuerpo, con la palma hacia arriba, No obstante, el brazo debe permanecer un poco elevado, permitiendo el paso de la cintra métrica.

- Rodee el brazo con el metro sobre el punto medio que fue marcado. Ajuste el metro sin comprimir los tejidos.

- Proceda a realizar la lectura. La medición antropométrica debe realizarse por lo menos dos veces, para verificar la precisión de la medida, si se encuentra variación superior a 0,2 cm debe proceder a repetirla.

- Registrar el resultado en la hoja de recogida de datos:

Circunferencia braquial (CB):

Menos de $21 \mathrm{~cm}$..........0,0 puntos

$21-22 \mathrm{~cm}$.....................0,5 puntos

Más de $22 \mathrm{~cm} \ldots \ldots \ldots . . . . . .1,0$ puntos

\section{Determinación circunferencia de la pantorrilla}

- Ubique la persona en posición supina con la rodilla flexionada formando un ángulo de 90 grados.

- Coloque la cinta métrica alrededor de la pantorrilla, deslícela hasta localizar la circunferencia máxima de la pantorrilla.

- Marque esta referencia antropométrica usando para ello un lápiz.

- Proceda a medirla sin comprimir los tejidos.

- Realice la lectura. La medición antropométrica debe realizarse por lo menos dos veces, para verificar la precisión de la medida, si se encuentra variación superior a 0,2 cm debe proceder a repetirla.

- Registrar el resultado en la hoja de recogida de datos

Circunferencia de la pantorrilla (CP):

Menos de $31 \mathrm{~cm}$. 0 puntos

Mayor o igual a $31 \mathrm{~cm}$ 1 puntos

\section{Control de cambios}

\begin{tabular}{|l|l|l|}
\hline Edición & Cambio realizado & Fecha \\
\hline & & \\
\hline & & \\
\hline & & \\
\hline
\end{tabular}


Anexo 6. Decálogo de la alimentación saludable en personas mayores de 65 años

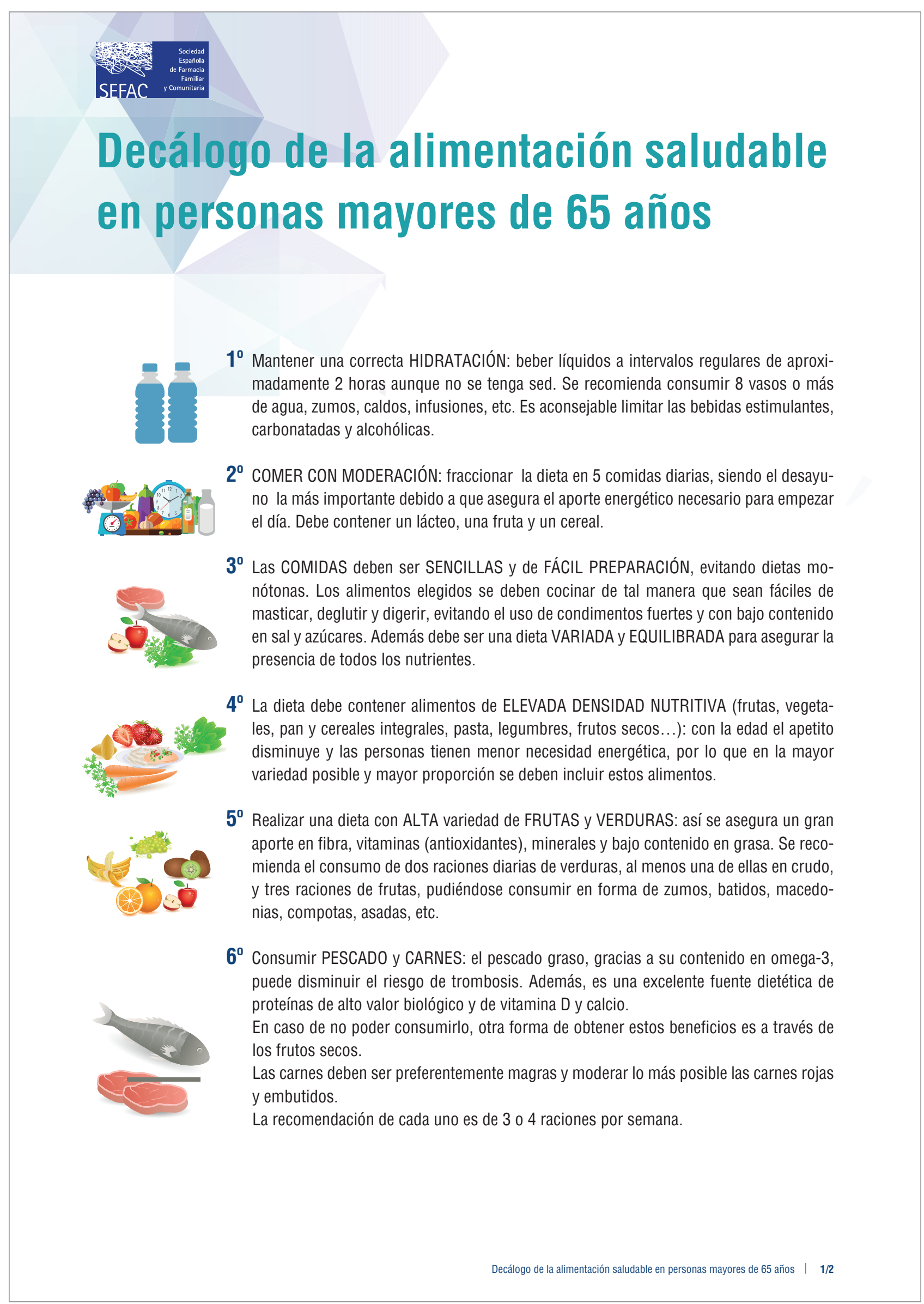




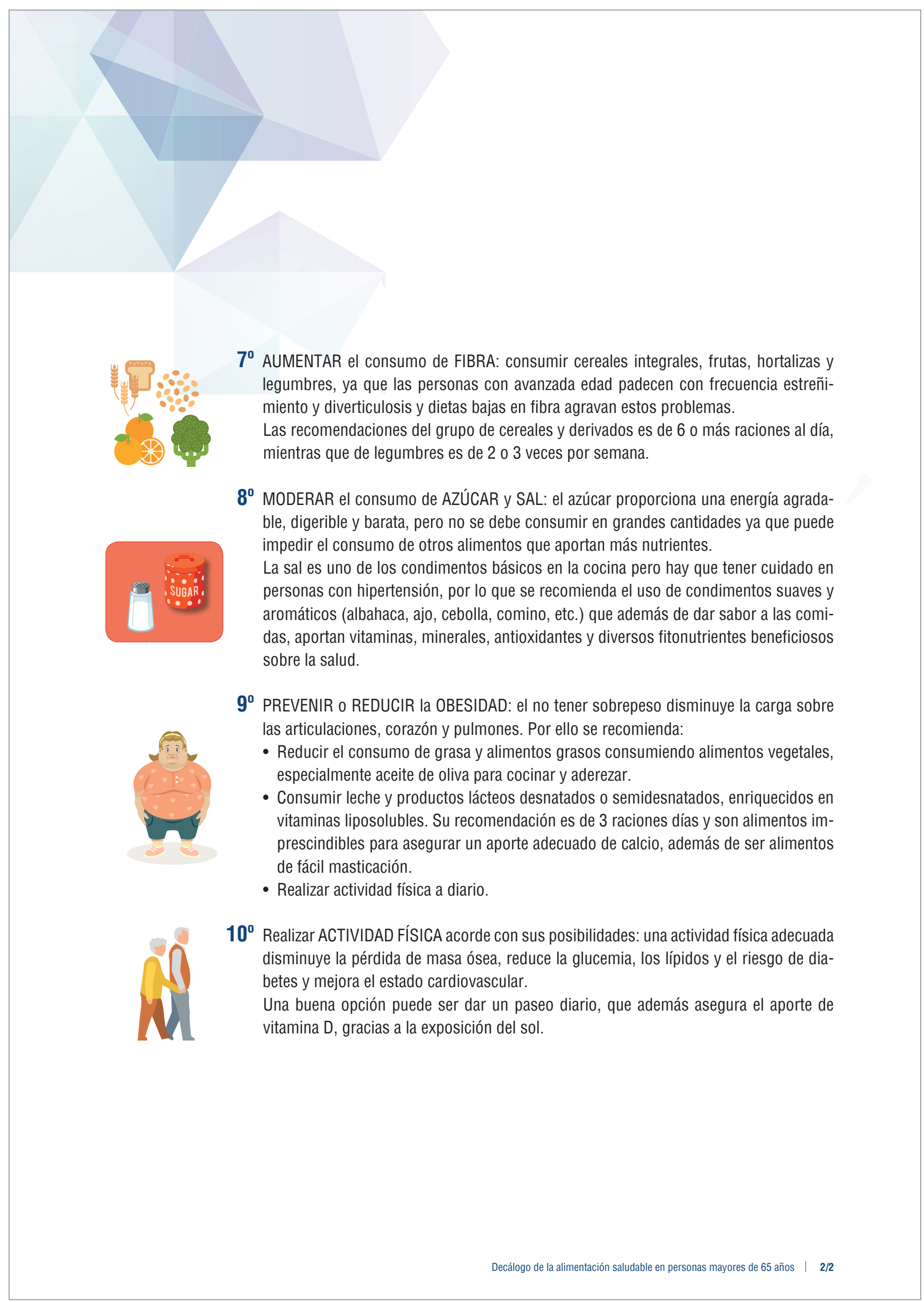


Anexo 7. Consejos de alimentación en diabetes

Recomendaciones de la Sociedad Española de Farmacia Familiar y Comunitaria a la población.

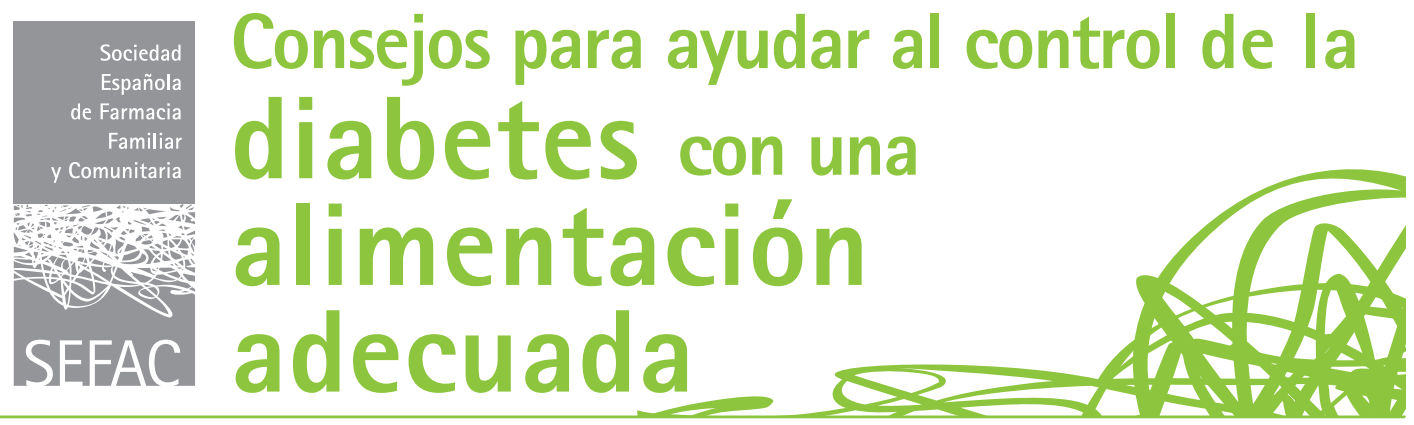

Un pillar fundamental

La alimentación equilibrada y el ejercicio adecuado a las necesidades de cada persona con diabetes, con el objetivo de conseguir un peso apropiado, son los pilares fundamentales para el tratamiento de esta patología, y se deben mantener aunque exista un tratamiento con medicamentos.

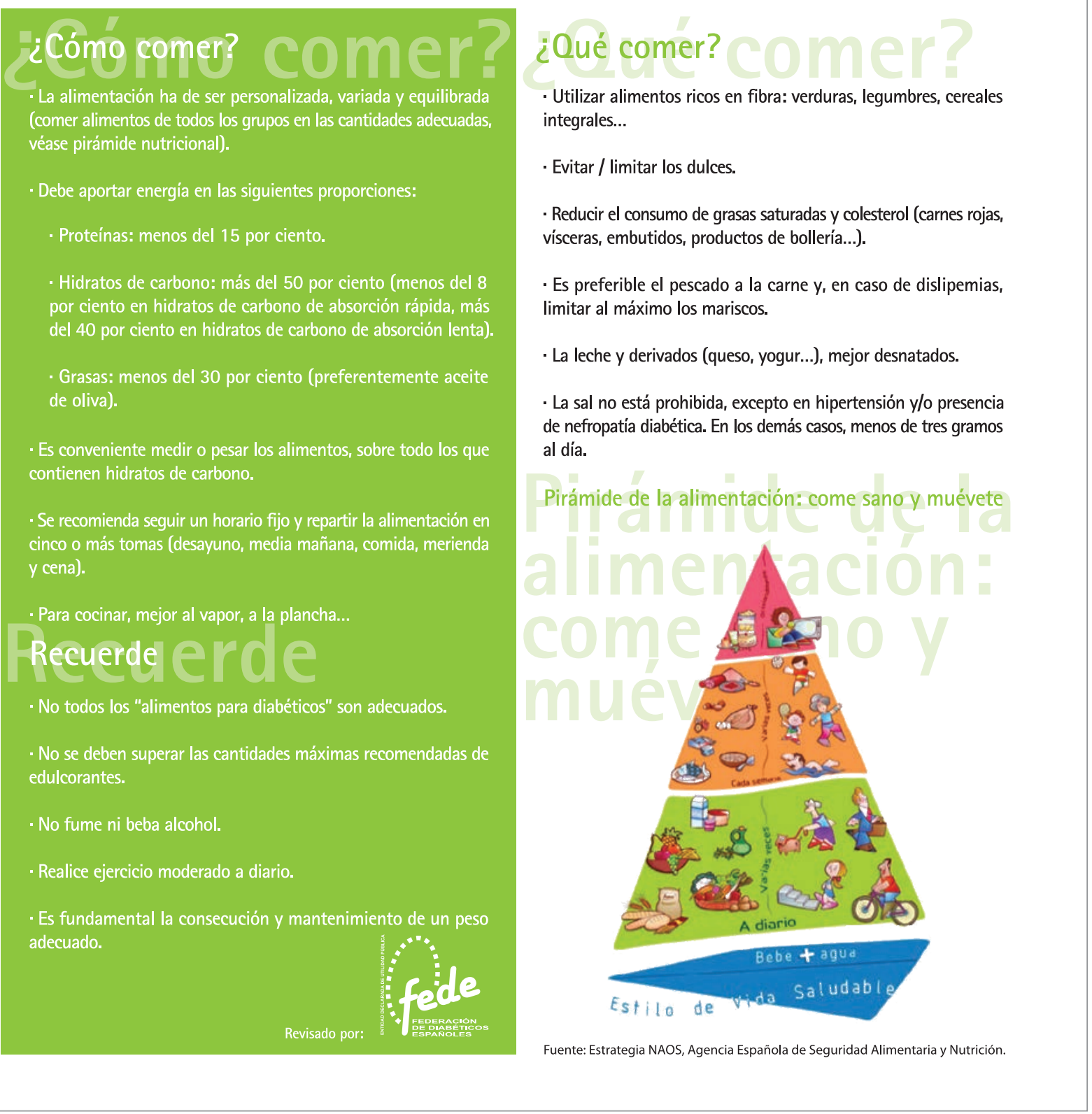

